\title{
Detector System for Three-Dimensional Imaging in the Variable Pressure/Environmental SEM
}

\author{
W. SŁóWKO* AND M. KRYSZTOF \\ Faculty of Microsystem Electronics and Photonics, Wrocław University of Technology \\ Z. Janiszewskiego 11/17, 50-372 Wrocław, Poland
}

\begin{abstract}
The authors developed a method of three-dimensional surface reconstruction, which takes advantage of the angular distribution of backscattered electron emission to obtain a digital map of surface elevations. Such quantitative information about the surface topography is obtained by digital processing of four input images acquired from four electron detectors. The method was adapted to environmental conditions by means of the vacuum-detector equipment applicable for a standard scanning electron microscopy. The main part of the equipment is the vacuum-detector head containing the intermediate vacuum chamber with a semiconductor backscattered electron detector inside. The detector captures a zenith backscattered electron fraction passing through the throttling aperture placed closely to the sample at the distance comparable to the aperture diameter $(0.6 \mathrm{~mm})$. The system was dedicated to the investigations of solid nonconductive samples at pressures $<10 \mathrm{hPa}$ but it has already been supplemented with a gaseous directional backscattered electron detector which can work at a distance of a few $\mathrm{mm}$ to be safe for biological samples.
\end{abstract}

DOI: $10.12693 /$ APhysPolA.123.877

PACS: 41.85.-p, 52.80.Dy

\section{Introduction}

Scanning electron microscopy (SEM) is a powerful tool in diagnostics and examination of semiconductors structures, micro-mechanical systems and biological specimens, however it still provides limited information about the third dimension of the surface topography. The authors developed a method of three-dimensional surface reconstruction, which takes advantage of the Lambert angular distribution of backscattered electron (BSE) emission to obtain a digital map of surface elevations. Such quantitative information about the surface topography is obtained by digital processing of four input images acquired from four electron detectors. This approach is often called a multi-detector method or a photometric method [1-3].

A three-dimensional reconstruction system designed by the authors as an attachment to SEM, consists of a quadruple directional electron detector, a frame-grabber and a PC-based processing unit. Software processing algorithms were developed to carry out the reconstruction process and compensate for several types of errors, inherent in the method [2]. Despite the complexity of the overall reconstruction algorithm it can operate very quickly, even in real-time for average SEM scanning rates. To obtain satisfying accuracy of the reconstructions special error compensation algorithms were developed. The presented method is particularly useful for the reconstruction of relatively smooth objects, without numerous surface details necessary for a more popular "stereoscopic

*corresponding author; e-mail: witold.slowko@pwr.wroc.pl method". Such smooth objects can usually be found among biological samples which have to be observed in water vapour atmosphere obtainable in the variable pressure and environmental SEM (VP/E SEM). This suggests that the method should be advantageously adapted to the VP/E SEM.

\section{Bases of the multidetector method}

The multidetector method is based on the Lambert angular distribution of backscattered and secondary electrons

$$
\delta\left(\phi_{\mathrm{p}}\right)=\delta_{0}\left(\cos \phi_{\mathrm{p}}\right)^{-n}, \quad n \approx 1 .
$$

A system of four detectors, placed symmetrically around the specimen (Fig. 1a), allows to extract information

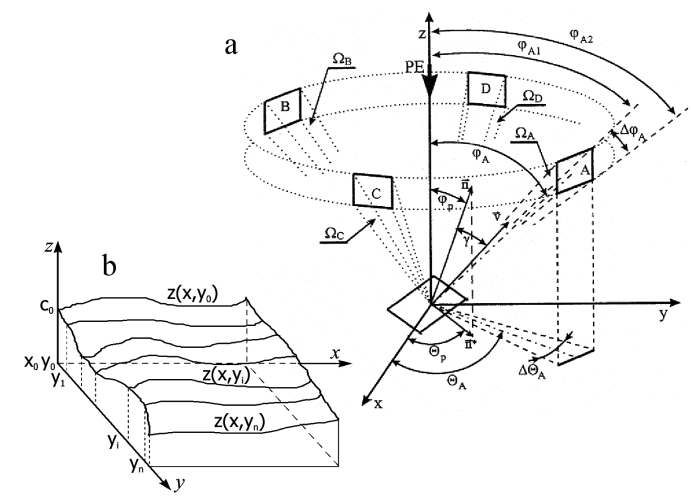

Fig. 1. Illustrations to the 3D reconstruction method: (a) four detector system in polar coordinates, (b) surface shape reconstruction according to Eq. (4). 
about local topography and composition. The expression for a detector signal can be written as follows:

$$
I_{A}=I_{\mathrm{PE}}\left(\delta_{0} / \pi\right)\left[d \tan \left(\phi_{\mathrm{p}}\right) \cos \left(\theta_{A}-\theta_{\mathrm{p}}\right)+c\right],
$$

where $\theta_{\mathrm{p}}, \phi_{\mathrm{p}}$ - local surface topography in polar coordinates, $\delta_{0}$ - secondary emission coefficient (depends on the material composition), $d_{A}, c_{A}, \theta_{A}$ - detector geometry coefficients, $I_{\mathrm{PE}}$ - electron beam current.

The local surface inclination may be obtained by dividing the difference by the sum of the opposite detector signals

$$
\frac{I_{A}-I_{B}}{I_{A}+I_{B}}=\frac{d}{c} \tan \phi_{\mathrm{p}} \cos \theta_{\mathrm{P}}=\frac{d}{c} \frac{\mathrm{d} z}{\mathrm{~d} x} .
$$

This provides a specimen topography profile if integrated along a scan line in the $x$ direction. The second pair of detectors gives the initial profile in the $y$ direction which allows to synthesize a fully $3 \mathrm{D}$ surface according to the expression beneath and Fig. 1b:

$$
\begin{gathered}
z(x, y)=a_{x} \int_{x_{0}}^{x}\left[\frac{I_{A}-I_{B}}{I_{A}+I_{B}}\right]_{y} \mathrm{~d} x \\
+a_{y} \int_{y_{0}}^{y}\left[\frac{I_{C}-I_{D}}{I_{C}+I_{D}}\right]_{x=x_{0}} \mathrm{~d} y .
\end{gathered}
$$

\section{Detector-vacuum system}

The VP/E SEM operates at elevated pressure in the sample chamber while the electron optical column is maintained at high vacuum. This implies differential pumping of the two regions which have to be separated by an intermediate chamber with vacuum of the order of $10^{-2} \mathrm{hPa}$, limited by two pinhole throttling apertures preventing intensive gas flow from the sample chamber to the high vacuum side $[4,5]$. The authors use here $1 \mathrm{hPa}$ $(100 \mathrm{~Pa})$ as the pressure unit as it is equal to $1 \mathrm{mbar}$ which still remains a popular unit among scientists.

A serious problem for the mentioned environmental adaptation is the directional detection system capable of working in gaseous atmosphere. The term directional detection means that each detector in the quadruple unit detects only electrons emitted from the sample in a strictly defined range of directions of the initial velocities which must not be changed by electron collisions with gas molecules. According to this criterion, BSEs showing an average energy about half the energy of the primary electron beam, should have the collision cross-section small enough to preserve initial directions of velocities at the detector distance. For instance, the mean free path at $1 \mathrm{hPa}$ of water vapour pressure and at the electron energy of $5 \mathrm{keV}$ is about $11 \mathrm{~mm}$ so the distance to the detector should be appropriately shorter to avoid false detection of scattered electrons.

Out of the known detector devices the semiconductor BSE detectors seem most suitable for the low vacuum conditions though their real size seems too large to fulfil the distance criterion at higher pressures. To overcome this problem the authors placed a four quadrant (4Q) PIN diode (playing a role of the quadruple BSE detector) in the intermediate chamber (Fig. 2) where the gas pressure is at least ten times lower than that in the sample chamber so electrons can cover a relatively long distance without scattering [6].

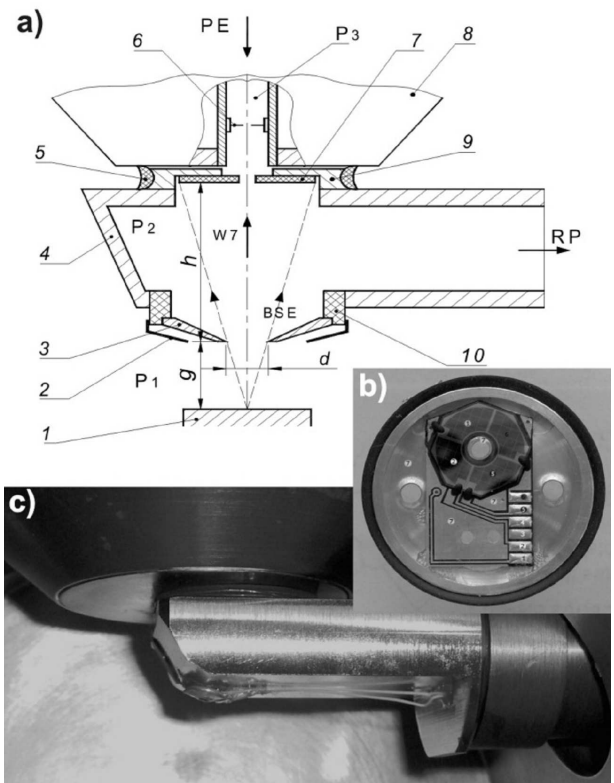

Fig. 2. BSE/SE detector system for 3D\&VP technology in a classic SEM: (a) scheme of the vacuum and detector head: 1 - sample stage, 2 - screening aperture, 3 - lower throttling aperture, 4 - intermediate vacuum chamber, 5 - ring gasket, 6 - upper throttling aperture, 7 - BSE detector, 8 - objective lens, $9-$ separating ring, $10-$ insulating ring, $P_{1}>P_{2}>P_{3}$ gas pressures, RP - rotary pump, $g, h$ - distances; (b) separating ring with four quadrant BSE detector; (c) intermediate vacuum and detector head positioned in the sample chamber.

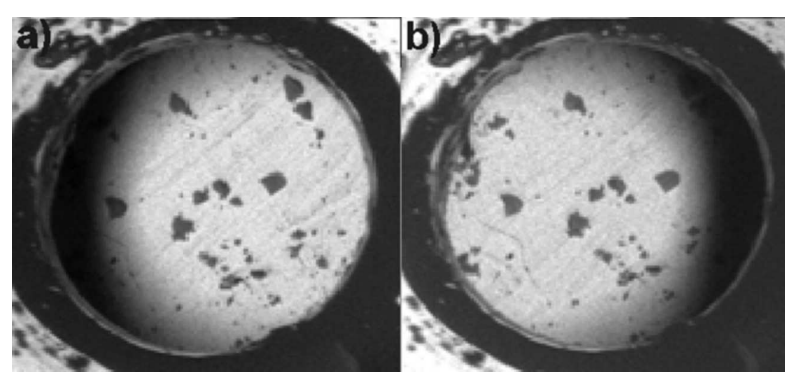

Fig. 3. Images of $\mathrm{Cu}-\mathrm{Be}$ foil seen through the throttling aperture of $0.6 \mathrm{~mm}$ in the diameter, at the distance $g=0.4 \mathrm{~mm}, P_{1}=0.5 \mathrm{hPa}$, air, obtained from the left (a) and right (b) BSE detector positioned on the $x$ axis.

A zenithal fraction of BSE generated from the sample surface enters the intermediate chamber through the lower throttling aperture of $0.6 \mathrm{~mm}$ in a diameter so its maximum distance $g$ to the sample for the detector (10 $\mathrm{mm}$ in a diameter) placed at $h=14 \mathrm{~mm}$ from the 
aperture, should be below $1 \mathrm{~mm}$ to keep the detector area within the BSE cone. It should be emphasized that at the mentioned maximum distance of $g=1 \mathrm{~mm}$ only BSE emitted from the central point of the view field creates a cone fitting exactly to the detector diameter and the rest is shadowed. For instance, the distance should be twice lower $(h=0.5 \mathrm{~mm})$ to obtain an unshadowed view field covering half of the throttling aperture diameter (Fig. 3).

\section{4. $3 \mathrm{D}$ reconstruction procedure in experiments}

Four quadrants (4Q) of the PIN diode playing a role of the BSE detector (Fig. 2b) produce four signals stored in the form of four input images, as in Fig. 4a. They are processed to obtain the $3 \mathrm{D}$ pattern of the surface shape

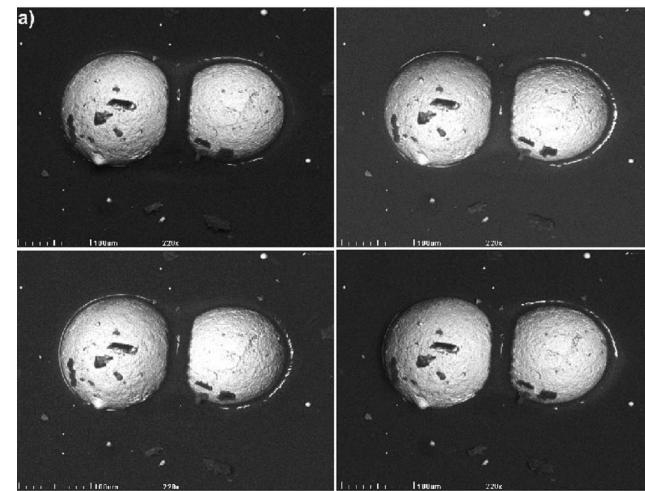

b)

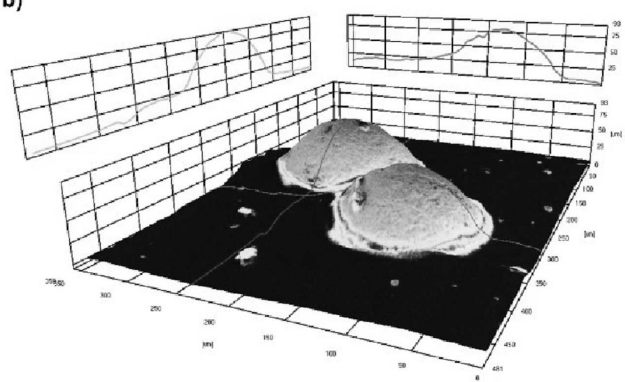

c)

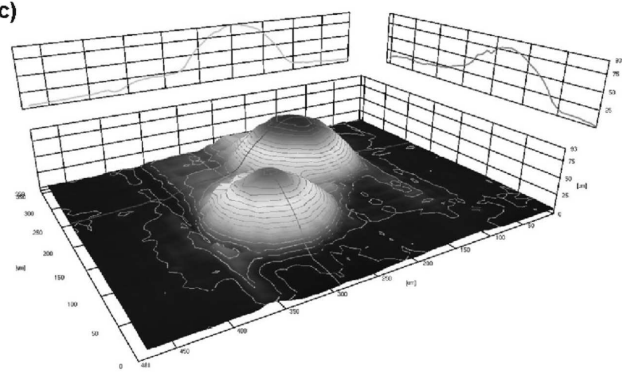

Fig. 4. Microballs on an adhesive tape: (a) four input BSE images for the 3D reconstruction, (b) axonometric view with surface profiles shown in the side grids and surface texture taken from the SE image with a halftone (originally colour) palette, (c) as above but with level lines and an elevation coding halftone (originally colour) palette instead texture. in the form of a bit-map which can be visualized in any desired form. For instance, it may be a perspective view with the surface profiles shown on the side grids. The shape can be expressed additionally by a height coding half tone (or colour) palette as in Fig. 4c. However, such surface shape is deprived of subtle details (mainly by the integration process occurring in the algorithm) usually present on every surface as the so-called texture. The source of the surface texture to be put on the shape may be one of the input images or advantageously an additional SE image. To this list of tested forms of the surface shape presentation, the anaglyph and roughness as well as waviness statistical parameters [3] may be added.

\section{Summary}

A classic high vacuum SEM equipped with the VP BSE detector system may become a good supplement for the standard instruments used for 3D imaging, as atomic force microscopy (AFM) or confocal laser scanning microscope (CLSM) for instance. The system has been designed as detachable equipment which does not imply any changes in an original structure of the microscope. Presently, the authors are carrying out vast investigations of the system and adapt it to wet biological samples. The wet sample surface is not as exactly defined as the solid one and should be positioned at a greater distance than the described system can accept. Thus, the authors designed a combined directional electron detector [7] supplemented with a BSE gaseous detector working at greater distances and higher pressures than the presented one.

\section{Acknowledgments}

Electronic equipment for the detector used in this work have been designed by Mr Jakub Brach. This work was supported with the budged resources for science in $2011 / 12$ as the research project.

\section{References}

[1] L. Reimer, R. Bongeler, V. Desai, Scan. Microsc. 1, 963 (1987).

[2] J. Paluszyński, W. Słówko, Vacuum 78, 533 (2005).

[3] J. Paluszyński, W. Słówko, J. Microsc. 233, 10 (2009).

[4] G.D. Danilatos, Adv. Electron. Electron Phys. 78, 1 (1990).

[5] D.J. Stokes, Principles and Practice of Variable Pressure/Environmental Scanning Electron Microscopy (VP-ESEM), Wiley, Chichester 2008.

[6] W. Słówko, Patent application PL396065, 2011.

[7] W. Słówko, Patent application PL399438, 2012. 